\title{
Guided wave phased array beamforming and imaging in composite plates
}

\author{
Lingyu Yu; Zhenhua Tian* \\ ${ }^{a}$ University of South Carolina, Department of Mechanical Engineering, Columbia SC
}

\begin{abstract}
This paper describes phased array beamforming using guided waves in anisotropic composite plates. A generic phased array algorithm is presented, in which direction dependent guided wave parameters and the energy skew effect are considered. This beamforming at an angular direction is achieved based on the classic delay-and-sum principle by applying phase delays to signals received at array elements and adding up the delayed signals. The phase delays are determined with the goal to maximize the array output at the desired direction and minimize it otherwise. For array characterization, the beam pattern of rectangular grid arrays in composite plates is derived. In addition to the beam pattern, the beamforming factor in terms of wavenumber distribution is defined to provide intrinsic explanations for phased array beamforming. The beamforming and damage detection in a composite plate are demonstrated using rectangular grid arrays made by a non-contact scanning laser Doppler vibrometer. Detection images of the composite plate with multiple surface defects at various directions are obtained. The results show that the guided wave phased array method is a potential effective method for rapid inspection of large composite structures.
\end{abstract}

Keywords: guided waves; phased arrays; anisotropic composite; array imaging; multiple defects; rapid inspection

\footnotetext{
* Correspondence author: tianz@email.sc.edu
} 


\section{INTRODUCTION}

Rapid inspection of large areas with nondestructive evaluation (NDE) methods is critical for ensuring operation ability and safety in aerospace industry, especially where safety related structural components are used [1,2]. Advanced composite materials have been increasingly used in aerospace industry. The Boeing 787 Dreamliner has an airframe comprising nearly 50\% carbon fiber reinforced plastic and other composites [3]. Though various NDE methods have been developed for metallic structures and proven effective, reliable and efficient evaluations for large composite structures are not yet well established [1,4]. Complexity of the advanced composite material manufacturing and in-service maintenance present challenges in evaluation tools and methods [4].

Ultrasonic NDE is the technique to provide an invasive means to inspect the condition of a component. Among various NDE methodologies, ultrasonic method is directly sensitive to mechanical changes and can be used to directly assess the mechanical condition and integrity of the composite structure [4]. However it is commonly considered that NDE using bulk waves is time-consuming since it requires point-by-point measurement over the inspected area and therefore is not efficient for large area inspection. To address this deficiency, the guided waves (GW) based ultrasonic techniques have been studied, developed, and demonstrated great potentials on metallic structures [5-8]. Compared to bulk waves, GW can travel long distances within the waveguides with low energy loss $[9,10]$. However, GW NDE is facing major challenges when being applied on composite structures [4]. The GW complexity caused by anisotropic and inhomogeneous properties in composite materials makes the traditional metalbased NDE methods inappropriate and sometimes even misleading [11-13]. 
When generating inspection results, an image of the structure being inspected often gives an efficient solution that quickly identifies and locates defects. Various imaging methods based on GW NDE have been explored including tomography [6, 14-16], sparse array [7, 17-24], synthetic aperture focusing technique [25-27], reconstruction algorithm for probabilistic inspection of damage (RAPID) [5, 28-30], and phased array [8, 31-64]. Among them, the phased array imaging is attractive since it uses sensors that are placed closed to each other in a compact format, steers the outputs of all sensors in a desired direction, and inspects the entire structure like a radar [31]. It hence allows for rapid inspection of large area with limited access. The additional advantages include reinforced wave energy in the steered direction, efficient and flexible control of the direction, improved signal-to-noise-ratio, and promising damage detection results [31].

Intensive study has been conducted on the GW phased array beamforming and damage detection on metallic plate like structures [8, 31-58]. A general beamforming algorithm for isotropic materials has been developed $[31,32]$ with investigation of beamforming optimization [33-36]. 1-D linear [37, 38] arrays and 2-D planar arrays [8, 31, 39] in various configurations have been designed and used for damage detection of hole or cracks with the arrays made of piezoelectric wafer sensors [31], piezoelectric paint sensors [39], or electromagnetic acoustic transducer (EMAT) [8].

Some researchers have started investigating the phased arrays for anisotropic composite materials [59-64]. Yan and Rose studied beamsteering of linear arrays in composite plates [59]. They found the traditional beamsteering technique for isotropic materials might fail in composite materials due to the anisotropic behaviors of the composite plates. Hence, they chose a quasi- 
isotropic wave mode for beamsteering, which can suppress the influence of the anisotropic behavior. Rajagopalan et al. adopted an array of a single transmitter and multiple receivers (STMR) to locate a defect (hole) in a composite plate [60]. In their array imaging method, they used a weakly anisotropic wave mode and assumed that the phase and group velocity directions coincide locally. Later, Vishnuvardhan et al. used the STMR array to detect impact induced delamination damage in a quasi-isotropic composite plate [61]. Leleux et al. used ultrasonic phased array probes for long range detection of defects in composite plates [62]. Their method was limited to wave modes having phase and group velocities oriented in the same direction, where the skew angle was zero. Purekar and Pines investigated the capability of 1-D linear phased arrays in detecting delamination damage in a cross-ply composite plate [63]. They showed the array can detect damage at $0^{\circ}$ direction, where the phase and group velocities had the same orientation. Osterc et al. investigated the beamsteering of 1-D linear arrays in composite laminates [64]. In their study, the exact phase velocity curve was used to develop a beamforming algorithm that accounted for non-omnidirectional guided wave propagation in anisotropic materials. It has found that compared to the array beamforming in isotropic plates, the array beamforming in anisotropic composite plates are more challenging. In anisotropic composite plates, guided wave parameters such as wavenumbers, phase velocities and group velocities are direction dependent due to the direction dependent physical properties of composite materials [11-13]. Moreover, the GW have energy skewness that the direction of group velocity is not always aligned to that of the phase velocity. Last but not least, the wave fronts of GW are no longer circular in composites. The traditional beamforming technique for isotropic materials may fail in composite materials due to the complexity involved with GW propagation. 
In this paper, we investigate $\mathrm{GW}$ beamforming in anisotropic laminated composite plates. Based on the classic delay-and-sum principle, a generic formula of phased array beamforming in anisotropic composite plates is developed, in which the direction dependent guided wave properties are adopted. The beamforming is demonstrated by implementation of 2-D rectangular grid arrays. Beamforming with various array configurations of rectangular grid arrays are investigated. For the proof of concept, laboratory tests are performed using rectangular grid arrays made of scanning points of a non-contact scanning laser Doppler vibrometer (SLDV) for detecting surface defects. The results show that multiple defects at various directions can be successfully detected and the phased array method can be useful for rapid inspection of large composite structures. The remainder of this paper is organized as follows: Section 2 presents the formulation of phased array beamforming in anisotropic composite plates; Section 3 presents beamforming characterization of 2-D rectangular grid arrays; Section 4 presents the implementation and detection of multiple defects in a composite plate using arrays made of scanning points of a non-contact SLDV. Section 5 concludes the paper with novelties, discussions and planned future work.

\section{GW BEAMFORMING IN COMPOSITE LAMINATES}

In this section, we formulate general GW phased array beamforming in anisotropic composite laminates based on the classic delay-and-sum principle.

\subsection{GW in composite laminates}

When a guided wave with frequency $\omega$ and wavenumber $\mathbf{k}$ is generated from a source at the coordinate origin $O$ in a composite plate, the wave arriving at the location $\mathbf{x}$ that is far away from the source (Figure 1a) can be expressed as [9, 10, 13], 


$$
u(t, \mathbf{x})=A e^{j(\omega t-\mathbf{k} \cdot \mathbf{x})}
$$

where $A$ is the amplitude, assuming independent of wave frequency. With the geometric relation illustrated in Figure 1a, we have,

$$
\mathbf{k} \cdot \mathbf{x}=|\mathbf{k}||\mathbf{x}| \cos \beta=k(\gamma)|\mathbf{x}| \cos \beta
$$

with $\beta$ being the angle between the wave propagation and wavenumber $\mathbf{k}$. Hence,

$$
u(t, \mathbf{x})=A e^{j[\omega t-k(\gamma)|\mathbf{x}| \cos \beta]}
$$

Using Eq.(1), for a source located at location $\mathbf{p}_{m}$, the wave resulted at the location $\mathbf{x}$ is,

$$
u_{m}(t, \mathbf{x})=A e^{j\left[\omega t-\mathbf{k} \cdot\left(\mathbf{x}-\mathbf{p}_{m}\right)\right]}
$$

In anisotropic composite laminates, GW parameters such as wavenumbers, phase velocities and group velocities are direction dependent, due to the direction dependent physical properties of composite materials [11-13]. Figure $1 \mathrm{~b}$ plots the wavenumber curve $k(\gamma)$ and slowness curve $k(\gamma) / \omega$. As illustrated in Figure $1 \mathrm{~b}$, the wavenumber vector $\mathbf{k}$ is perpendicular to the wave front and the group velocity vector $\mathbf{c}_{\mathrm{g}}$ is orthogonal to the wavenumber curve $k(\gamma)$ [11-13]. The angle $\gamma$ of the wavenumber vector $\mathbf{k}$ is referred to as wavenumber angle. The angle $\theta$ of the group velocity vector $\mathbf{c}_{\mathrm{g}}$ is referred to as group velocity angle (or energy propagation angle). The angle $\beta$ between wavenumber angle $\gamma$ and energy propagation angle $\theta$ is referred to as skew angle, with the relation $\beta=\gamma-\theta$. It can be seen when $\mathbf{c}_{\mathrm{g}}$ is not parallel to $\mathbf{k}$, the skew angle $\beta$ is not zero and hence the wave energy propagation direction is not perpendicular to the wave front.

\subsection{Delay-and-sum beamforming}

Consider an array with $M$ identical elements located at $\left\{\mathbf{p}_{m}\right\}(m=0,1,2, \ldots M-1)$ which are geometrically close to each other. The phase center is defined as the origin $O$ of the Cartesian 
coordinate system, i.e., $1 / M \sum_{m=0}^{M-1} \mathbf{p}_{m}=\mathbf{0}$. Each element serves as a wave source. When all elements generate waves with frequency $\omega$ and wavenumber vector $\mathbf{k}$ simultaneously, using Eq. (4) the total output (synthesized wave) of the array at location $\mathbf{x}$ can be derived as,

$$
z(t, \mathbf{x})=\sum_{m=0}^{M-1} A e^{j\left[\omega t-\mathbf{k} \cdot\left(\mathbf{x}-\mathbf{p}_{m}\right)\right]}=u(t, \mathbf{x}) \sum_{m=0}^{M-1} e^{j \mathbf{k} \cdot \mathbf{p}_{m}}
$$

It is seen from Eq. (5) that the synthesized wave $z(t, \mathbf{x})$ is an amplification of the wave $u(t, \mathbf{x})$ emitted from the Origin. The amplification is controlled by the exponential component $\sum_{m=0}^{M-1} e^{j \mathbf{k} \cdot \mathbf{p}_{m}}$ in Eq. (5). Therefore, by adjusting the component $\sum_{m=0}^{M-1} e^{j \mathbf{k} \cdot \mathbf{p}_{m}}$, we can control the amplification. One way to adjust the component $\sum_{m=0}^{M-1} e^{j \mathbf{k} \cdot \mathbf{p}_{m}}$ is applying phase delays to all exponents. For example, to maximize the amplification $\sum_{m=0}^{M-1} e^{j \mathbf{k} \cdot \mathbf{p}_{m}}$ in a specific direction $\theta_{S}$, we can apply phase delays $\Delta_{m}\left(\theta_{S}\right)$ to exponents and let all exponents be zero,

$$
\sum_{m=0}^{M-1} e^{j\left[\mathbf{k} \cdot \mathbf{p}_{m}-\Delta_{m}\left(\theta_{S}\right)\right]}=\sum_{m=0}^{M-1} e^{j 0}=M, \text { where } \Delta_{m}\left(\theta_{S}\right)=\mathbf{k} \cdot \mathbf{p}_{m}
$$

Using phase delays $\Delta_{m}\left(\theta_{S}\right)$, the amplification is maximized at the direction $\theta_{S}$, i.e., generating a directional "beam". $\theta_{S}$ is also known as steering angle. Eq. (6) shows phase delays $\Delta_{m}\left(\theta_{S}\right)$ depend on the $m^{\text {th }}$ element's position vector $\mathbf{p}_{m}$ and wavenumber vector $\mathbf{k}$.

In anisotropic composites, the wavenumber vector $\mathbf{k}$ depends on wave frequency $\omega$ and wavenumber angle $\gamma_{s}$. In addition, the geometry relation between wavenumber angle $\gamma_{s}$ and steering angle $\theta_{S}$ is $\gamma_{S}=\theta_{S}+\beta_{S}$, where $\beta_{S}$ is referred to as skew angle. Hence, phase delays $\Delta_{m}\left(\theta_{S}\right)$ in Eq.(6) can be further expressed as,

$$
\Delta_{m}\left(\theta_{S}\right)=\mathbf{k}\left(\omega, \theta_{S}+\beta_{S}\right) \cdot \mathbf{p}_{m}
$$


It can be seen from Eq.(7) the phase delay includes a frequency dependent term, $\mathbf{k}\left(\omega, \theta_{S}+\beta_{S}\right)$. Hence, it is frequency dependent and has the advantage of compensating dispersion effect (a.k.a. frequency dependent wave properties) by using frequency-related components during the delaying $[8,66]$. This is otherwise not readily achievable in the commonly used time delay methods [31, 37, 59].

In addition to phase delays, weighting factors $w_{m}$ can also be applied to delayed waves to further control the quality of beamforming [9, 65]. In a summary, with phase delays and weighting factors, the beamforming is represented as,

$$
z(t, \mathbf{x})=u(t, \mathbf{x}) \sum_{m=0}^{M-1} w_{m} e^{j\left[\mathbf{k} \cdot \mathbf{p}_{m}-\Delta_{m}\left(\theta_{S}\right)\right]}=u(t, \mathbf{x}) \sum_{m=0}^{M-1} w_{m} e^{j\left[\mathbf{k}-\mathbf{k}\left(\omega, \theta_{S}+\beta_{S}\right)\right] \cdot \mathbf{p}_{m}}
$$

\section{CHARACTERIZATION OF RECTANGULAR GRID ARRAYS}

This section investigates 2-D rectangular grid arrays in composite plates. For array characterization, beamforming factor in terms of wavenumber and beam pattern in terms of wave propagation direction are used. With the beamforming factor and beam pattern, beamforming of rectangular grid arrays with different configurations are characterized and investigated in details.

\subsection{Rectangular grid arrays}

Elements in phased arrays can be arranged with various configurations, such as linear [37], rectangular [31], or spiral [39] arrangements. In this study, rectangular grid arrays with elements being uniformly placed on rectangular grids are investigated. Figure 2 illustrates a $P \times Q$ rectangular grid array (total number of elements: $M=P \times Q$ ) with its phase center as the coordinate origin $O$. The coordinates of the $(p, q)^{\text {th }}(p=0,1,2, \ldots P-1$ and $q=0,1,2, \ldots Q-1)$ element in the array are, 


$$
\mathbf{p}_{p, q}=\left(\left(p-\frac{P-1}{2}\right) d_{x},\left(q-\frac{Q-1}{2}\right) d_{y}\right)
$$

where $d_{x}$ and $d_{y}$ are array spacings in $x$ and $y$ directions, respectively. The array spans in $x$ and $y$ directions then are given as,

$$
D_{x}=(P-1) d_{x} \text { and } D_{y}=(Q-1) d_{y}
$$

The beamforming is implemented on an 8-ply [0/45/90/-45]s layup carbon fiber reinforced polymer (CFRP) composite plate (material properties in Table 1). The $\mathrm{A}_{0}$ Lamb mode at 120 $\mathrm{kHz}$ is used for the beamforming. Figure $3 \mathrm{a}, 3 \mathrm{~b}$ and $3 \mathrm{c}$ plot the wavenumber, slowness and phase velocity curves derived by using the semi-analytical finite element (SAFE) method [67]. The maximum wavenumber components in $k_{x}$ and $k_{y}$ directions are $k_{\mathrm{x}, \max }=0.55 \mathrm{rad} / \mathrm{mm}$ and $k_{\mathrm{y}, \max }=$ $0.68 \mathrm{rad} / \mathrm{mm}$, resulting in the minimum wavelengths $\lambda_{\mathrm{x}, \min }=2 \pi / k_{\mathrm{x}, \max }=11.4 \mathrm{~mm}$ and $\lambda_{\mathrm{y}, \min }=$ $2 \pi / k_{\mathrm{y}, \max }=9.2 \mathrm{~mm}$

To study the beamforming, three array configurations (A1, A2, A3) listed in Table 2 are considered. Among them, (a) A1 and A2 have the same spacing, while the spans in A2 are twice of those in A1; (b) A2 and A3 have the same span, while the spacings in A3 are twice of those in A2.

\subsection{Characterization of rectangular grid arrays}

For beamforming characterization, the beamforming factor $(B F)[9,31]$ is adopted, which is given as,

$$
B F=\frac{1}{M} \sum_{m=0}^{M-1} w_{m} e^{j\left[\mathbf{k} \cdot \mathbf{p}_{m}-\Delta_{m}\left(\theta_{S}\right)\right]}
$$

Substituting Eq. (7) into Eq. (11), the beamforming factor becomes, 


$$
B F\left(\mathbf{k} \mid w_{m}, \theta_{S}\right)=\frac{1}{M} \sum_{m=0}^{M-1} w_{m} e^{j\left[\mathbf{k}-\mathbf{k}\left(\omega, \theta_{S}+\beta_{S}\right)\right] \cdot \mathbf{p}_{m}}
$$

The beamforming factor in Eq. (12) can be interpreted as a function of wavenumber vector $\mathbf{k}$, denoted as $B F\left(\mathbf{k} \mid w_{m}, \theta_{S}\right)$ in the wavenumber domain. $\theta_{S}$ and $w_{m}$ represent two parameters that can control the beamforming direction and beam shape. For $2-\mathrm{D} \mathrm{GW}, B F\left(\mathbf{k} \mid w_{m}, \theta_{S}\right)$ evaluates the beamforming result at any wavenumber vector $\mathbf{k}$ in the $k_{x}-k_{y}$ wavenumber plane.

Besides $B F\left(\mathbf{k} \mid w_{m}, \theta_{S}\right)$, a directional beam pattern is also introduced, which is a function of the wave propagation direction $\theta$ as,

$$
B F\left(\theta \mid w_{m}, \theta_{S}\right)=\frac{1}{M} \sum_{m=0}^{M-1} w_{m} e^{j\left[\mathbf{k}(\omega, \theta+\beta)-\mathbf{k}\left(\omega, \theta_{S}+\beta_{S}\right)\right] \cdot \mathbf{p}_{m}}
$$

where $\mathbf{k}(\omega, \theta+\beta)$ is the wavenumber dispersion relation of $\mathrm{GW}$. The directional beam pattern $B F\left(\theta \mid w_{m}, \theta_{S}\right)$ evaluates the beamforming output w.r.t. the wave propagation direction $\theta$.

\subsubsection{Characterization of rectangular grid arrays using $B F\left(\mathbf{k} \mid w_{m}, \theta_{S}\right)$}

Beamforming of three rectangular grid arrays in Table 2 is investigated by the beamforming factor $B F\left(\mathbf{k} \mid w_{m}, \theta_{S}\right)$ given in Eq.(12). By substituting element coordinates in Eq.(9) into Eq.(12), we can derive $B F\left(\mathbf{k} \mid w_{p, q}, \theta_{S}\right)$ for rectangular grid arrays,

$$
B F\left(\mathbf{k} \mid w_{p, q}, \theta_{S}\right)=\frac{1}{P \cdot Q} \sum_{p=0}^{P-1} \sum_{q=0}^{Q-1} w_{p, q} e^{j\left[\mathbf{k}-\mathbf{k}\left(\omega, \theta_{S}+\beta_{S}\right)\right] \cdot\left(\left(p-\frac{P-1}{2}\right) d_{x},\left(q-\frac{Q-1}{2}\right) d_{y}\right)}
$$

By substituting array spans in Eq.(10) into Eq.(14), $B F\left(\mathbf{k} \mid w_{p, q}, \theta_{S}\right)$ becomes,

$$
B F\left(\mathbf{k} \mid w_{p, q}, \theta_{S}\right)=\frac{1}{\left(\frac{D_{x}}{d_{x}}+1\right)\left(\frac{D_{y}}{d_{y}}+1\right)} \sum_{p=0}^{\frac{D_{x}}{d_{x}}} \sum_{q=0}^{\frac{D_{y}}{d_{y}}} w_{p, q} e^{j\left[\mathbf{k}-\mathbf{k}\left(\omega, \theta_{S}+\beta_{S}\right)\right] \cdot\left(p d_{x}-\frac{D_{x}}{2}, q d_{y}-\frac{D_{y}}{2}\right)}
$$


This equation shows the beamforming factor $B F\left(\mathbf{k} \mid w_{p, q}, \theta_{S}\right)$ also depends on the weighting factor $w_{p, q}$, steering angle $\theta_{S}$, and array configuration parameters $d_{x}, d_{y}, D_{x}$ and $D_{y}$.

In this study, uniform weighting $\left(w_{p, q}=1\right)$ is used. Figure 4 a plots $B F\left(\mathbf{k} \mid w_{p, q}=1\right.$, no) of array A1 without applying delays as an intensity image in the $k_{x}-k_{y}$ wavenumber plane. Four highlighted spots at $(0,0),\left(0,4 k_{\mathrm{y}, \max }\right),\left(4 k_{\mathrm{y}, \max }, 0\right)$ and $\left(4 k_{\mathrm{y}, \max }, 4 k_{\mathrm{y}, \max }\right)$ are present in the given region. These spots represent the local maxima of $B F\left(\mathbf{k} \mid w_{p, q}=1\right.$, no). When the array generates GW with wavenumbers at these local maxima, the array's output will be optimized. The sizes of spots are evaluated by the full width at one-half peak value (denoted as $F W H M_{x}$ and $F W H M_{y}$ for $k_{x}$ and $k_{y}$ directions, respectively) [65], given in Table 3. Smaller FWHM signifies higher resolution as well as better directionality.

Note that Figure $4 \mathrm{a}$ only plots $B F\left(\mathbf{k} \mid w_{p, q}=1\right.$, no $)$ in the wavenumber range $-1.0 \mathrm{rad} / \mathrm{mm}$ $\leq k_{x} \leq 3.7 \mathrm{rad} / \mathrm{mm}$ and $-1.0 \mathrm{rad} / \mathrm{mm} \leq k_{y} \leq 3.7 \mathrm{rad} / \mathrm{mm}$. Indeed highlighted spots are present in a periodical pattern within the entire wavenumber domain. The periodical pattern can be perceived from the expression of beamforming factor in Eq.(15). The periods $K_{x}$ and $K_{y}$ of the periodical pattern in $k_{x}$ and $k_{y}$ directions are $2 \pi / d_{x}$ and $2 \pi / d_{y}$, respectively.

A wavenumber curve $k(\gamma)$ of the $120 \mathrm{kHz} \mathrm{A}_{0}$ mode in the subject composite plate is also plotted in Figure 4a. It can be seen that no maxima of $B F\left(\mathbf{k} \mid w_{p, q}=1\right.$, no $)$ falls on the wavenumber curve of the adopted wave mode. This indicates that if the array generates the 120 $\mathrm{kHz} \mathrm{A}_{0}$ mode without delaying, the array will not have maximized output.

In order to achieve maximized array output, phase delays are applied to relocate certain maxima on the wavenumber curve. Figure $4 \mathrm{~b}$ plots $B F\left(\mathbf{k} \mid w_{p, q}=1, \theta_{S}=90^{\circ}\right)$ of array A1 with 
phase delays of $(-0.02,0.68) \cdot \mathbf{p}_{p, q}$. The delays are selected such that the local maxima located at $(0,0)$ before the delays moves to the point $(-0.02,0.68) \mathrm{rad} / \mathrm{mm}$ on the wavenumber curve.

Figure 5a and $5 \mathrm{~b}$ plot $B F\left(\mathbf{k} \mid w_{p, q}=1, \theta_{S}=90^{\circ}\right)$ images of arrays A2 and A3, when phase delays $(-0.02,0.68) \cdot \mathbf{p}_{p, q}$ are applied. $F W H M$ values (in Table 3) of arrays A2 and A3 (with the same span) are the same and both smaller than those of array A1 (with a smaller span). This indicates the larger array span gives the smaller $F W H M$ value.

Wavenumber periods of arrays A1, A2 and A3 are also derived, as listed in Table 3. The periods of arrays A1 and A2 (with the same array spacing) are the same, and both larger than those of array A3 (with a larger array spacing). Therefore, the smaller array spacing gives the larger wavenumber period.

The wavenumber period can affect the beamforming performance. For example, for the array A3, the $B F\left(\mathbf{k} \mid w_{p, q}=1, \theta_{S}=90^{\circ}\right)$ image in Figure 5b shows there are two intensified spots at $(-0.02,0.68) \mathrm{rad} / \mathrm{mm}$ and $(-0.02,-0.68) \mathrm{rad} / \mathrm{mm}$ on the wavenumber curve $k(\gamma)$ of the $120 \mathrm{kHz}$ $\mathrm{A}_{0}$ mode. This means if array A3 generates a $120 \mathrm{kHz} \mathrm{A}_{0}$ mode with phase delays (-0.02, 0.68) $\mathbf{p}_{p, q}$, the synthesized waves generated from the array will have two intensified components: (1) waves with the wavenumber $(-0.02,0.68) \mathrm{rad} / \mathrm{mm}$, and (2) waves with the wavenumber ($0.02,-0.68) \mathrm{rad} / \mathrm{mm}$, contradicting the single beam intention. Hence situations with more than one intensified components should be avoided since they give misleading beamforming results. Therefore, wavenumber periods should satisfy $K_{x}>2 k_{\mathrm{x}, \max }$ and $K_{y}>2 k_{\mathrm{y}, \max }$, i.e., the array spacings should satisfy $d_{x}<\lambda_{\mathrm{x}, \min } / 2$ and $d_{y}<\lambda_{\mathrm{y}, \min } / 2$. 


\subsubsection{Characterization of rectangular grid arrays using $B F\left(\theta \mid w_{m}, \theta_{S}\right)$}

The beamforming can also be characterized in terms of the directional beam pattern $B F\left(\theta \mid w_{m}, \theta_{S}\right)$ given in Eq. (13). By substituting element coordinates in Eq.(9) into Eq.(13), we can derive,

$$
B F\left(\theta \mid w_{p, q}, \theta_{S}\right)=\frac{1}{P Q} \sum_{p=0}^{P-1} \sum_{q=0}^{Q-1} w_{p, q} e^{j\left[\mathbf{k}(\omega, \theta+\beta)-\mathbf{k}\left(\omega, \theta_{S}+\beta_{S}\right)\right] \cdot\left(\left(p-\frac{P-1}{2}\right) d_{x},\left(q-\frac{Q-1}{2}\right) d_{y}\right)}
$$

Or using array span and spacing as,

$$
B F\left(\theta \mid w_{p, q}, \theta_{S}\right)=\frac{1}{\left(\frac{D_{x}}{d_{x}}+1\right)\left(\frac{D_{y}}{d_{y}}+1\right)} \sum_{p=0}^{\frac{D_{x}}{d_{x}}} \sum_{q=0}^{\frac{D_{y}}{d_{y}}} w_{p, q} e^{j\left[\mathbf{k}(\omega, \theta+\beta)-\mathbf{k}\left(\omega, \theta_{S}+\beta_{S}\right)\right] \cdot\left(p d_{x}-\frac{D_{x}}{2}, q d_{y}-\frac{D_{y}}{2}\right)}
$$

Eq.(17) shows the beam pattern $B F\left(\theta \mid w_{p, q}, \theta_{S}\right)$ also depends on the weighting factor $w_{p, q}$, steering angle $\theta_{S}$, and array configuration parameters $d_{x}, d_{y}, D_{x}$ and $D_{y}$.

Figure 6a plots the directional beam pattern $B F\left(\theta \mid w_{p, q}=1\right.$, no $)$ of array A1 using the 120 $\mathrm{kHz} \mathrm{A}_{0}$ mode without applying phase delays. The amplitude is seen low at all directions. This is consistent with $B F\left(\mathbf{k} \mid w_{p, q}=1\right.$, no) image in Figure 4a, where on maxima is on the dispersion curve of $\mathrm{A}_{0}$ mode. To maximize the amplitude to a desired direction $\theta_{S}$, phase delays are applied. Figure $6 \mathrm{~b}$ plots beamsteering results toward $0^{\circ}, 45^{\circ}, 90^{\circ}$ and $135^{\circ}$ directions, by applying delays of $(0.55,-0.02) \cdot \mathbf{p}_{p, q},(0.37,0.51) \cdot \mathbf{p}_{p, q},(-0.02,0.68) \cdot \mathbf{p}_{p, q}$ and $(-0.37,0.54) \cdot \mathbf{p}_{p, q}$, respectively.

To evaluate beamforming qualities at different directions, $F W H M$ values of array A1 are determined as given in Table 4, where the $0^{\circ}$ beamforming has the smallest $F W H M$ value, thus, the best resolution among the four directions. 
Directional beam patterns of arrays A2 and $\mathrm{A} 3$ at $\theta_{S}=0^{\circ}, 45^{\circ}, 90^{\circ}$ and $135^{\circ}$ have also been studied as given in Figure $7 \mathrm{a}$ and $7 \mathrm{~b}$. For $0^{\circ}, 45^{\circ}$ and $135^{\circ}$ beamsteering angles, beam patterns of array A2 are nearly the same as those of array A3. However, for the $90^{\circ}$ beamsteering direction, the beam pattern of array A3 in Figure $7 \mathrm{~b}$ (dotted line) has an additional lobe at $265^{\circ}$ with nearly the same amplitude and shape as the mainlobe at $90^{\circ}$. This spurious mainlobe (also known as grating lobe $[9,65])$ is induced by large element spacing. The appearance of grating lobe in Figure $7 \mathrm{~b}$ can also be confirmed in the $B F\left(\mathbf{k} \mid w_{p, q}=1, \theta_{S}=90^{\circ}\right.$ ) image (Figure 5b) where an additional intensified spot at $(-0.02,-0.68) \mathrm{rad} / \mathrm{mm}$ shows on the wavenumber curve.

The $F W H M$ values of all arrays are also derived and compared in Table 4. FWHM values of arrays A2 and A3 are the same while smaller than those of array A1. Hence, arrays A2 and A3 offer better resolutions than array A1.

\section{SLDV GW PHASED ARRAY}

In this section, guided wave beamforming and damage detection in a laminated composite plate using rectangular grid arrays are implemented. A hybrid measurement system consisting of a surface bonded PZT wafer and a non-contact scanning laser Doppler vibrometer (SLDV) is used for GW actuation and sensing [68]. Phased arrays are constructed by selected points of a small SLDV scanning area. Using selected scanning points of the SLDV scan gives the flexibility of constructing arrays of various configurations with only one scan. The element spacing in SLDV arrays can be smaller than $0.1 \mathrm{~mm}$ [68], while the spacing in traditional arrays such as piezoelectric wafer arrays is usually limited by the sensor size. Four quartz rods are surface bonded on a composite plate at various angular positions as defects to be detected by the phased array method. 


\subsection{Experimental setup}

Figure 8 shows the experimental setup of the GW sensing in a composite plate. The test specimen is an 8-ply [0/45/90/-45] $]_{\mathrm{S}}$ layup CFRP composite plate with dimensions of $610 \mathrm{~mm} \times$ $610 \mathrm{~mm} \times 2.54 \mathrm{~mm}$. The material properties are listed in Table 1. A PZT wafer (APC 851: $7 \mathrm{~mm}$ diameter, $0.2 \mathrm{~mm}$ thickness) is installed to generate $\mathrm{GW}$. The PZT center is set as the coordinate origin. GW are excited by a 3-cycle toneburst at $120 \mathrm{kHz}$ generated from a function generator (model: Agilent 33522B) and amplified to 30V by a voltage amplifier (model: Krohn-Hite 1506). Four identical defects are simulated by bonding quartz rods of $10 \mathrm{~mm}$ high and $8 \mathrm{~mm}$ diameter $\left(\mathrm{Q}_{1}, \mathrm{Q}_{2}, \mathrm{Q}_{3}\right.$ and $\left.\mathrm{Q}_{4}\right)$ on the plate surface at different angles $0^{\circ}, 45^{\circ}, 90^{\circ}$ and $135^{\circ}$, as shown in Figure 8b. All rods are placed $100 \mathrm{~mm}$ away from the coordinate origin.

An SLDV (model: Polytec PSV-400-M2) is used to acquire the velocity wavefield of GW over a small scanning area $(45 \mathrm{~mm} \times 45 \mathrm{~mm}$ square) centered at the coordinate origin from the back side of the plate. From the small scanning area, selected scanning points are used to construct phased arrays for damage detection. The horizontal and vertical spatial resolutions of the scanning are both $0.1 \mathrm{~mm}$. Based on the Doppler effect, the SLDV measures the GW velocity $v(t, \mathbf{x})$ along the laser beam over the scanning area, as a function of both time $t$ and space $\mathbf{x}$. In the test, the laser beam is set normal to the plate such that the out-of-plane velocity is acquired.

\subsection{SLDV measurements}

Figure $9 \mathrm{a}$ and $9 \mathrm{~b}$ plot wavefields in the $45 \mathrm{~mm} \times 45 \mathrm{~mm}$ scanning area measured by the SLDV, at $30 \mu$ s showing incident waves generated from the actuator and at $145 \mu$ s showing reflection waves from the four defects, respectively. For identifying the wave mode, wavenumber spectra of these wavefields are obtained by frequency-wavenumber analysis as detailed in [68-70]. The 
theoretical wavenumber curve of $\mathrm{A}_{0}$ mode derived by using the SAFE method is provided to confirm the existence of $\mathrm{A}_{0}$ wave mode. Both spectra show their wavenumber components are on the curve of $\mathrm{A}_{0}$ mode. The comparisons in Figure $9 \mathrm{c}$ and $9 \mathrm{~d}$ verify the theoretical wavenumber curve derived by SAFE method. Moreover, the comparison confirms that both incident and reflection waves are $\mathrm{A}_{0}$ mode.

\subsection{Array beamsteering and imaging}

The phased array is constructed using SLDV scanning points at selected locations $\left\{\mathbf{p}_{m}\right\} \quad(m=0$, $1,2, \ldots M-1)$, whose phase center satisfies $1 / M \sum_{m=0}^{M-1} \mathbf{p}_{m}=\mathbf{0}$. From the time-space wavefield $v(t, \mathbf{x})$ acquired by the SLDV, the signal at the $\mathrm{m}^{\text {th }}$ array point $\left(\mathbf{p}_{m}\right)$ can be denoted as $v_{m}(t)=v\left(t, \mathbf{p}_{\mathrm{m}}\right)$. Its frequency spectrum can be derived using the Fourier transform, as:

$$
V_{m}(\omega)=\mathcal{F}\left[v_{m}(t)\right]=\int_{-\infty}^{\infty} v_{m}(t) e^{-j \omega t} d t
$$

Using the frequency spectrum $V_{m}(\omega)$, we can derive the beamforming of the array in frequencyspace representation $Z(\omega, \mathbf{x})$ :

$$
Z(\omega, \mathbf{x})=\sum_{m=0}^{M-1} w_{m} V_{m}(\omega) e^{j\left[-\varphi(\omega, \mathbf{x})-\Delta_{m}(\omega, \mathbf{x})\right]}
$$

where,

$$
\Delta_{m}(\omega, \mathbf{x})=\mathbf{k}(\omega, \gamma) \cdot \mathbf{p}_{m} \text {, and } \varphi(\omega, \mathbf{x})=-2 \mathbf{k}(\omega, \gamma) \cdot \mathbf{x}
$$

$\Delta_{m}(\omega, \mathbf{x})$ is the phase delay applied to the $\mathrm{m}^{\text {th }}$ array point for beamsteering. $\varphi(\omega, \mathbf{x})$ represents the spatial phase shift. As guided waves travel from the PZT to the damage and then back to the array, they undergo a spatial phase shift $\varphi(\omega, \mathbf{x})$. Thus, $-\varphi(\omega, \mathbf{x})$ is applied in Eq. (19) in order to compensate such a spatial phase shift. The spatial phase shift $\varphi(\omega, \mathbf{x})$ and the phased delay $\Delta_{m}(\omega$, $\mathbf{x})$ both include the frequency dependent term $\mathbf{k}(\omega, \gamma)$. Hence, by considering the frequency 
dependence, the dispersion effect (frequency dependent wave properties) can then be taken into consideration.

Using inverse Fourier transform, the frequency-space representation $Z(\omega, \mathbf{x})$ is transformed back to the time-space domain, as:

$$
z(t, \mathbf{x})=\mathcal{F}^{-1}[Z(\omega, \mathbf{x})]=\frac{1}{2 \pi} \int_{-\infty}^{\infty} Z(\omega, \mathbf{x}) e^{j \omega t} d \omega
$$

where $z(t, \mathbf{x})$ represents the array beamforming in time-space representation. An inspection image of the plate is then acquired at $z(t=0, \mathbf{x})$ or building an intensity image by defining the pixel value at location $\mathbf{x}$ as:

$$
I(\mathbf{x})=|z(t=0, \mathbf{x})|
$$

\subsection{Array imaging results}

From the wavefields measured in the scanning area by the SLDV, multiple scanning points can be selected to construct phased arrays. The selected scanning points are serving as sensing elements of phased arrays. By this means, we can construct phased arrays of different configurations by using scanning points at different locations.

The array performance is highly related to the array configuration, such as array spacing and number of elements, as presented previous work [31, 37]. For example, the array spacing is usually set at a half wavelength of the selected guided wave [31,37]. With the same spacing, a larger number of elements gives better array performance though longer data acquisition time is needed [31]. With these considerations, three phased arrays (A1, A2, and A3 listed in Table 2) with different configurations (spacings and numbers of elements) are investigated, to show how guided wave phased array beamforming is affected by array configurations. Among them, (a) A1 
and A2 have the same spacing, while the A2 has more elements than A1; (b) A2 and A3 have the same number of elements, while the spacing in A3 is twice of that in A2. The beamforming results of these three arrays have been theoretically studied in subsection 3.2. In this section, the three arrays are experimentally investigated.

From the $45 \mathrm{~mm} \times 45 \mathrm{~mm}$ scanning area, $21 \times 21$ points are chosen to construct the array A2 (its configuration is given in Table 2). Using the phased array method presented in subsection 3.2, a synthesized time-space wavefield $z(t, \mathbf{x})$ is constructed. The synthesized wavefield $z(t=0, \mathbf{x})$ at $0 \mu$ s (in Figure 10a) shows four reconstructed wave packets in $0^{\circ}, 45^{\circ}, 90^{\circ}$ and $135^{\circ}$ directions.

Using the pixel definition given in Eq. (22), an intensity image of the plate is reconstructed using the synthesized wavefield $z(t=0, \mathbf{x})$, as shown in Figure 10b. It clearly indicates the presence of four defects at various directions as by presenting four highlighted areas in the image, with localization errors less than $5 \mathrm{~mm}$. Please note the damage at $0^{\circ}$ has the highest magnitude which is due to the fact that the guided waves have strongest propagation along this direction in the subject composite plate.

Figure 11a and $11 \mathrm{~b}$ also give intensity images generated by arrays A1 and A3. By comparing the imaging results of arrays A1, A2 and A3 in Figures 11a, 10b and 11b, it can be found that arrays A2 and A3 give four intensified areas (indicating the four defects) with smaller sizes than array A1. It means the imaging results of arrays A2 and A3 have better resolution than array A1. This is consistent with the $B F\left(\theta \mid w_{m}, \theta_{S}\right)$ comparison in subsection 3.2, which shows that $B F\left(\theta \mid w_{m}, \theta_{S}\right)$ plots of arrays $\mathrm{A} 2$ and $\mathrm{A} 3$ have smaller $F W H M$ and better angular resolution than array A1. Although arrays A2 and A3 nearly give the same imaging result at the four defects, the imaging result of array A3 shows an additional intensified area around the 
location $(-10,-100) \mathrm{mm}$. This additional area is induced by the spatial aliasing, and consistent with the $B F\left(\theta \mid w_{m}, \theta_{S}\right)$ plot of the array A3 (in Figure 7b) which shows a grating lobe at $265^{\circ}$ direction.

\section{CONCLUSIONS}

In this paper, we have presented a generic guided wave phased array beamforming method for anisotropic composite laminates. The method is implemented with a non-contact SLDV guided wave sensing system and successfully detects multiple defects at various locations. Investigation on effects of array configurations on beamforming and damage detection has also been conducted.

The novelty of this work is multifold. First, the generic beamforming formula uniquely considers the direction-dependent guided wave properties and the energy skew effect; thus it allows for phased array beamforming in anisotropic composites without taking any quasiisotropic assumption. Second, 2D phased array beamforming characterization in frequency domain has been conducted. The phase delay in frequency domain offers the advantage of compensating the guided wave dispersion effect through the use of frequency-dependent wavenumber. Third, the phased array method is validated through a non-contact SLDV system which allows for the flexibility of using selected points from the SLDV scan to construct phase arrays of various configurations. The high spatial resolution feature of the SLDV system also significantly reduces array element spacing that gives the potential of using guided waves with smaller wavelengths to detect smaller defects in composite structures. Last, we have shown that the present method can detect multiple defects at various locations in the composite plate, which is less studied in the literature to the best knowledge of the authors. 
Despite the success of phased array beamforming and multiple defects detection in a composite plate presented here, further studies are still needed. Besides the selected $120 \mathrm{kHz} \mathrm{A0}$ mode used in the current study, additional work needs to be done with guided waves at other frequencies or different wave modes in highly anisotropic composite plates. The effectiveness of the present method should be also evaluated with realistic defects such as impact induced delamination in composite plates. In addition, direction dependent guided wave attenuation in the composite plates should be investigated and considered in the beamforming. The potential of weighting factors to improve the beamforming quality could also be investigated.

\section{REFERENCES}

[1] Giurgiutiu, V. and Soutis, C., "Enhanced Composites Integrity through Structural Health Monitoring," Applied Composite Materials, vol. 19, pp. 813-829, 2012.

[2] Staszewski, W. J., Mahzan, S., and Traynor, R., "Health Monitoring of Aerospace Composite Structures - Active and Passive Approach," Composites Science and Technology, vol. 69, pp. 16781685, Sep 2009.

[3] Hale, J., "Boeing 787 from the Ground Up," AERO, vol. QTR_04, pp. 17-23, 2006.

[4] Rose, J. L., "Successes and Challenges in Ultrasonic Guided Waves for Ndt and Shm," in National Seminar \& Exhibition on Non-Destructive Evaluation, Tiruchirappalli, India, 2009, pp. 1-11.

[5] Zhao, X. L., Qian, T., Mei, G., Kwan, C., Zane, R., Walsh, C., Paing, T., and Popovic, Z., "Active Health Monitoring of an Aircraft Wing with an Embedded Piezoelectric Sensor/Actuator Network: Ii. Wireless Approaches," Smart Materials \& Structures, vol. 16, pp. 1218-1225, Aug 2007.

[6] Leonard, K. R. and Hinders, M. K., "Lamb Wave Tomography of Pipe-Like Structures," Ultrasonics, vol. 43, pp. 574-583, Jun 2005.

[7] Michaels, J. E. and Michaels, T. E., "Guided Wave Signal Processing and Image Fusion for in Situ Damage Localization in Plates," Wave Motion, vol. 44, pp. 482-492, Jun 2007.

[8] Wilcox, P. D., "Omni-Directional Guided Wave Transducer Arrays for the Rapid Inspection of Large Areas of Plate Structures," Ieee Transactions on Ultrasonics Ferroelectrics and Frequency Control, vol. 50, pp. 699-709, Jun 2003.

[9] Giurgiutiu, V., Structural Health Monitoring with Piezoelectric Wafer Active Sensors. Boston, MA: Academic Press, 2008.

[10] Rose, J. L., Ultrasonic Waves in Solid Media. New York: Cambridge University Press, 1999.

[11] Wang, L. and Yuan, F. G., "Group Velocity and Characteristic Wave Curves of Lamb Waves in Composites: Modeling and Experiments," Composites Science and Technology, vol. 67, pp. 13701384, Jun 2007.

[12] Glushkov, E., Glushkova, N., and Eremin, A., "Group Velocity of Cylindrical Guided Waves in Anisotropic Laminate Composites," Journal of Acoustic Society of America, vol. 135, pp. 148-154, 2014. 
[13] Nayfeh, A. H., Wave Propagation in Layered Anisotropic Media. Amsterdam, The Netherlands: Elsevier, 1995.

[14] Huthwaite, P. and Simonetti, F., "High-Resolution Guided Wave Tomography," Wave Motion, vol. 50, pp. 979-993, Jul 2013.

[15] Prasad, S. M., Balasubramaniam, K., and Krishnamurthy, C. V., "Structural Health Monitoring of Composite Structures Using Lamb Wave Tomography," Smart Materials \& Structures, vol. 13, pp. N73-N79, Oct 2004.

[16] Koduru, J. P. and Rose, J. L., "Mode Controlled Guided Wave Tomography Using Annular Array Transducers for Shm of Water Loaded Plate Like Structures," Smart Materials and Structures, vol. 22, p. 125021, Dec 2013.

[17] Chen, X., Michaels, J. E., and Michaels, T. E., "Design of Distributed Sparse Arrays for Lamb Wave Shm Based Upon Estimated Scattering Matrices," AIP Conf. Proc., vol. 1581, pp. 248-255, 2014.

[18] Yu, L. and Leckey, C., "Lamb Wave-Based Quantitative Crack Detection Using a Focusing Array Algorithm," Journal of Intelligent Material Systems and Structures, vol. 24, pp. 1138-1152, 2012.

[19] Levine, R. M. and Michaels, J. E., "Model-Based Imaging of Damage with Lamb Waves Via Sparse Reconstruction," Journal of Acoustic Society of America, vol. 133, pp. 1525-1534, 2013.

[20] Hall, J. S. and Michaels, J. E., "Minimum Variance Ultrasonic Imaging Applied to an in Situ Sparse Guided Wave Array," Ieee Transactions on Ultrasonics Ferroelectrics and Frequency Control, vol. 57, pp. 2311-2323, Oct 2010.

[21] Clarke, T., Cawley, P., Wilcox, P. D., and Croxford, A. J., "Evaluation of the Damage Detection Capability of a Sparse-Array Guided-Wave Shm System Applied to a Complex Structure under Varying Thermal Conditions," Ieee Transactions on Ultrasonics Ferroelectrics and Frequency Control, vol. 56, pp. 2666-2678, Dec 2009.

[22] Michaels, J. E., "Detection, Localization and Characterization of Damage in Plates with an in Situ Array of Spatially Distributed Ultrasonic Sensors," Smart Materials \& Structures, vol. 17, p. 035035, Jun 2008.

[23] Wang, C. H., Rose, J. T., and Chang, F. K., "A Synthetic Time-Reversal Imaging Method for Structural Health Monitoring," Smart Materials \& Structures, vol. 13, pp. 415-423, Apr 2004.

[24] Ng, C. T. and Veidt, M., "A Lamb-Wave-Based Technique for Damage Detection in Composite Laminates," Smart Materials \& Structures, vol. 18, p. 074006, Jul 2009.

[25] Stepinski, T., "An Implementation of Synthetic Aperture Focusing Technique in Frequency Domain," Ieee Transactions on Ultrasonics Ferroelectrics and Frequency Control, vol. 54, pp. 1399-1408, Jul 2007.

[26] Sicard, R., Goyette, J., and Zellouf, D., "A Saft Algorithm for Lamb Wave Imaging of Isotropic Plate-Like Structures," Ultrasonics, vol. 39, pp. 487-494, Apr 2002.

[27] Sicard, R., Chahbaz, A., and Goyette, J., "Guided Lamb Waves and L-Saft Processing Technique for Enhanced Detection and Imaging of Corrosion Defects in Plates with Small Depth-toWavelength Ratio," Ieee Transactions on Ultrasonics Ferroelectrics and Frequency Control, vol. 51, pp. 1287-1297, Oct 2004.

[28] Hay, T. R., Royer, R. L., Gao, H. D., Zhao, X., and Rose, J. L., "A Comparison of Embedded Sensor Lamb Wave Ultrasonic Tomography Approaches for Material Loss Detection," Smart Materials \& Structures, vol. 15, pp. 946-951, Aug 2006.

[29] Van Velsor, J. K., Gao, H., and Rose, J. L., "Guided-Wave Tomographic Imaging of Defects in Pipe Using a Probabilistic Reconstruction Algorithm," Insight, vol. 49, pp. 532-537, Sep 2007.

[30] Zhao, X. L., Gao, H. D., Zhang, G. F., Ayhan, B., Yan, F., Kwan, C., and Rose, J. L., "Active Health Monitoring of an Aircraft Wing with Embedded Piezoelectric Sensor/Actuator Network: I. 
Defect Detection, Localization and Growth Monitoring," Smart Materials \& Structures, vol. 16, pp. 1208-1217, Aug 2007.

[31] Yu, L. and Giurgiutiu, V., "In Situ 2-D Piezoelectric Wafer Active Sensors Arrays for Guided Wave Damage Detection," Ultrasonics, vol. 48, pp. 117-134, Apr 2008.

[32] Ambrozinski, L., Stepinski, T., and Uhl, T., "Efficient Tool for Designing 2d Phased Arrays in Lamb Waves Imaging of Isotropic Structures," Journal of Intelligent Material Systems and Structures, p. online, 2014.

[33] Engholm, M. and Stepinski, T., "Direction of Arrival Estimation of Lamb Waves Using Circular Arrays," Structural Health Monitoring-an International Journal, vol. 10, pp. 467-480, Sep 2011.

[34] de Marchi, L., Moll, J., and Marzani, A., "A Sparsity Promoting Algorithm for Time of Flight Estimation in Guided Waves-Based Shm," in EWSHM-7th European Workshop on Structural Health Monitoring, Nantes, France, 2014, pp. 583-590.

[35] Engholm, M., Stepinski, T., and Olofsson, T., "Imaging and Suppression of Lamb Modes Using Adaptive Beamforming," Smart Materials and Structures, vol. 20, p. 085024, Aug 2011.

[36] Kwon, H. S., Lee, S. S., and Kim, J. Y., "Beam Pattern Improvement by Compensating Array Nonuniformities in a Guided Wave Phased Array," Smart Materials and Structures, vol. 22, p. 085002 , Aug 2013.

[37] Giurgiutiu, V. and Bao, J., "Embedded-Ultrasonics Structural Radar for in Situ Structural Health Monitoring of Thin-Wall Structures," Structural Health Monitoring-an International Journal, vol. 3, pp. 121-140, Jun 2004.

[38] Deutsch, W. A. K., Cheng, A., and Achenbach, J. D., "Self-Focusing of Rayleigh Waves and Lamb Waves with a Linear Phased Array," Research in Nondestructive Evaluation, vol. 9, pp. 81-95, 1997.

[39] Yoo, B., Purekar, A. S., Zhang, Y., and Pines, D. J., "Piezoelectric-Paint-Based Two-Dimensional Phased Sensor Arrays for Structural Health Monitoring of Thin Panels," Smart Materials \& Structures, vol. 19, p. 075017, 2010.

[40] Fromme, P., Wilcox, P. D., Lowe, M. J. S., and Canvley, P., "On the Development and Testing of a Guided Ultrasonic Wave Array for Structural Integrity Monitoring," Ieee Transactions on Ultrasonics Ferroelectrics and Frequency Control, vol. 53, pp. 777-785, Apr 2006.

[41] Giridhara, G., Rathod, V. T., Naik, S., Roy Mahapatra, D., and Gopalakrishnan, S., "Rapid Localization of Damage Using a Circular Sensor Array and Lamb Wave Based Triangulation," Mechanical Systems and Signal Processing, vol. 24, pp. 2929-2946, 2010.

[42] Jarmer, G. J. S., Flynn, E. B., and Todd, M. D., "Multi-Wave-Mode, Multi-Frequency Detectors for Guided Wave Interrogation of Plate Structures," Structural Health Monitoring-an International Journal, vol. 13, pp. 120-130, Mar 2014.

[43] Kannajosyula, H., Lissenden, C. J., and Rose, J. L., "Analysis of Annular Phased Array Transducers for Ultrasonic Guided Wave Mode Control," Smart Materials and Structures, vol. 22, p. 085019, Aug 2013.

[44] Kim, D. and Philen, M., "Guided Wave Beamsteering Using Mfc Phased Arrays for Structural Health Monitoring: Analysis and Experiment," Journal of Intelligent Material Systems and Structures, vol. 21, pp. 1011-1024, Jul 2010.

[45] Malinowski, P., Wandowski, T., Trendafilova, I., and Ostachowicz, W., "A Phased Array-Based Method for Damage Detection and Localization in Thin Plates," Structural Health Monitoring-an International Journal, vol. 8, pp. 5-15, Jan 2009.

[46] Ostachowicz, W., Kudela, P., Malinowski, P., and Wandowski, T., "Damage Localisation in PlateLike Structures Based on Pzt Sensors," Mechanical Systems and Signal Processing, vol. 23, pp. 1805-1829, Aug 2009. 
[47] Prado, V. T., Higuti, R. T., Kitano, C., Martinez-Graullera, O., and Adamowski, J. C., "Lamb Mode Diversity Imaging for Non-Destructive Testing of Plate-Likestructures," Ndt \& E International, vol. 59, pp. 86-95, 2013.

[48] Purekar, A. S., Pines, D. J., Sundararaman, S., and Adams, D. E., "Directional Piezoelectric Phased Array Filters for Detecting Damage in Isotropic Plates," Smart Materials \& Structures, vol. 13, pp. 838-850, Aug 2004.

[49] Rajagopalan, J., Balasubramaniam, K., and Krishnamurthy, C. V., "A Single Transmitter MultiReceiver (Stmr) Pzt Array for Guided Ultrasonic Wave Based Structural Health Monitoring of Large Isotropic Plate Structures," Smart Materials \& Structures, vol. 15, pp. 1190-1196, Oct 2006.

[50] Romanoni, M., Gonella, S., Apetre, N., and Ruzzene, M., "Two-Dimensional Periodic Actuators for Frequency-Based Beam Steering," Smart Materials \& Structures, vol. 18, p. 125023, Dec 2009.

[51] Senesi, M., Xu, B., and Ruzzene, M., "Experimental Characterization of Periodic FrequencySteerable Arrays for Structural Health Monitoring," Smart Materials \& Structures, vol. 19, p. 055026, 2010.

[52] Seung, H. M., Kim, H. W., and Kim, Y. Y., "Development of an Omni-Directional ShearHorizontal Wave Magnetostrictive Patch Transducer for Plates," Ultrasonics, vol. 53, pp. 13041308, Sep 2013.

[53] Sundararaman, S., Adams, D. E., and Rigas, E. J., "Biologically Inspired Structural Diagnostics through Beamforming with Phased Transducer Arrays," International Journal of Engineering Science, vol. 43, pp. 756-778, May 2005.

[54] Swift, C. I., Pierce, S. G., and Culshaw, B., "Generation of a Steerable Ultrasonic Beam Using a Phased Array of Low Power Semiconductor Laser Sources and Fiber Optic Delivery," Smart Materials \& Structures, vol. 16, pp. 728-732, Jun 2007.

[55] Velichko, A. and Wilcox, P. D., "Guided Wave Arrays for High Resolution Inspection," Journal of the Acoustical Society of America, vol. 123, pp. 186-196, Jan 2008.

[56] Wilcox, P. D., Lowe, M., and Cawley, P., "Omnidirectional Guided Wave Inspection of Large Metallic Plate Structures Using an Emat Array," Ieee Transactions on Ultrasonics Ferroelectrics and Frequency Control, vol. 52, pp. 653-665, Apr 2005.

[57] Higuti, R. T., Martinez-Graullera, O., Martin, C. J., Octavio, A., Elvira, L., and de Espinosa, F. M., "Damage Characterization Using Guided-Wave Linear Arrays and Image Compounding Techniques," Ieee Transactions on Ultrasonics Ferroelectrics and Frequency Control, vol. 57, pp. 1985-1995, Sep 2010.

[58] Koduru, J. P., Momeni, S., and Rose, J. L., "Phased Annular Array Transducers for Omnidirectional Guided Wave Mode Control in Isotropic Plate Like Structures," Smart Materials and Structures, vol. 22, p. 125022, Dec 2013.

[59] Yan, F. and Rose, J. L., "Guided Wave Phased Array Beam Steering in Composite Plates," Health Monitoring of Structural and Biological Systems 2007, vol. 6532, pp. G5320-G5320, 2007.

[60] Rajagopalan, J., Multila, K. H. M. O. A., Balasubramaniam, K., and Krishnamurthy, C. V., "A Phase Reconstruction Algorithm for Lamb Wave Based Structural Health Monitoring of Anisotropic Multilayered Composite Plates," Journal of the Acoustical Society of America, vol. 119, pp. 872-878, Feb 2006.

[61] Vishnuvardhan, J., Muralidharan, A., Krishnamurthy, C. V., and Balasubramaniam, K., "Structural Health Monitoring of Anisotropic Plates Using Ultrasonic Guided Wave Stmr Array Patches," Ndt \& E International, vol. 42, pp. 193-198, Apr 2009.

[62] Leleux, A., Micheau, P., and Castaings, M., "Long Range Detection of Defects in Composite Plates Using Lamb Waves Generated and Detected by Ultrasonic Phased Array Probes," Journal of Nondestructive Evaluation, vol. 32, pp. 200-214, Jun 2013. 
[63] Purekar, A. S. and Pines, D. J., "Damage Detection in Thin Composite Laminates Using Piezoelectric Phased Sensor Arrays and Guided Lamb Wave Interrogation," Journal of Intelligent Material Systems and Structures, vol. 21, pp. 995-1010, 2010.

[64] Osterc, P., Kim, D., and Yoo, B., "Phased Array Beamsteering in Composite Laminates for Guided Wave Structural Health Monitoring," presented at the ASME 2013 Conference on Smart Materials, Adaptive Structures and Intelligent Systems, Snowbird, Utah, USA, 2013.

[65] Johnson, D. H. and Dudgeon, D. E., Array Signal Processing: Concepts and Techniques. Upper Saddle River, New Jersey, USA: Prentice-Hall Inc., 1993.

[66] Wilcox, P. D., "A Rapid Signal Processing Technique to Remove the Effect of Dispersion from Guided Wave Signals," Ieee Transactions on Ultrasonics Ferroelectrics and Frequency Control, vol. 50, pp. 419-427, Apr 2003.

[67] Bartoli, I., Marzani, A., di Scalea, F. L., and Viola, E., "Modeling Wave Propagation in Damped Waveguides of Arbitrary Cross-Section," Journal of Sound and Vibration, vol. 295, pp. 685-707, Aug 222006.

[68] Yu, L. and Tian, Z., "Lamb Wave Structural Health Monitoring Using a Hybrid Pzt-Laser Vibrometer Approach," Structural Health Monitoring, vol. 12, pp. 469-483, 2013.

[69] Tian, Z. and Yu, L., "Lamb Wave Frequency-Wavenumber Analysis and Decomposition," Journal of Intelligent Material Systems and Structures, vol. 25, pp. 1107-1123, 2014.

[70] Michaels, T. E., Michaels, J. E., and Ruzzene, M., "Frequency-Wavenumber Domain Analysis of Guided Wavefields," Ultrasonics, vol. 51, pp. 452-466, 2011. 
(a)

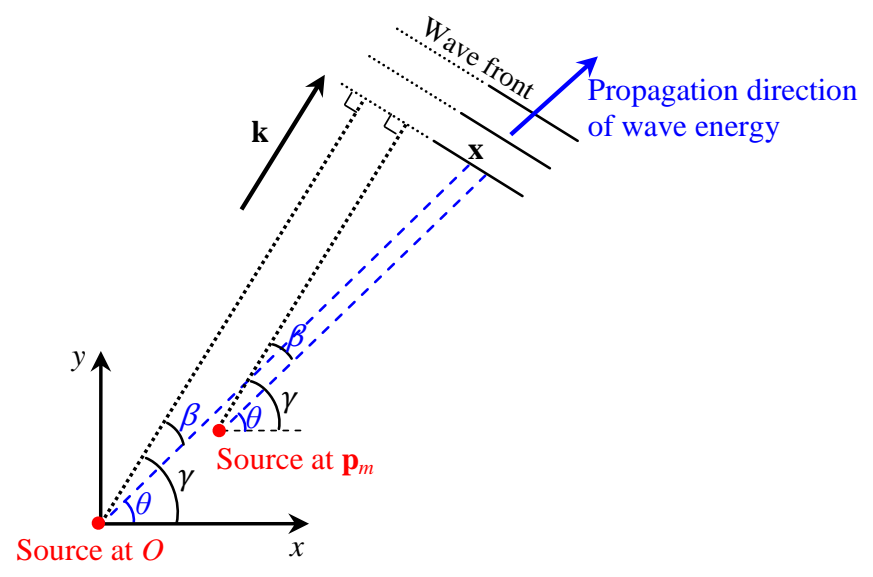

(b)
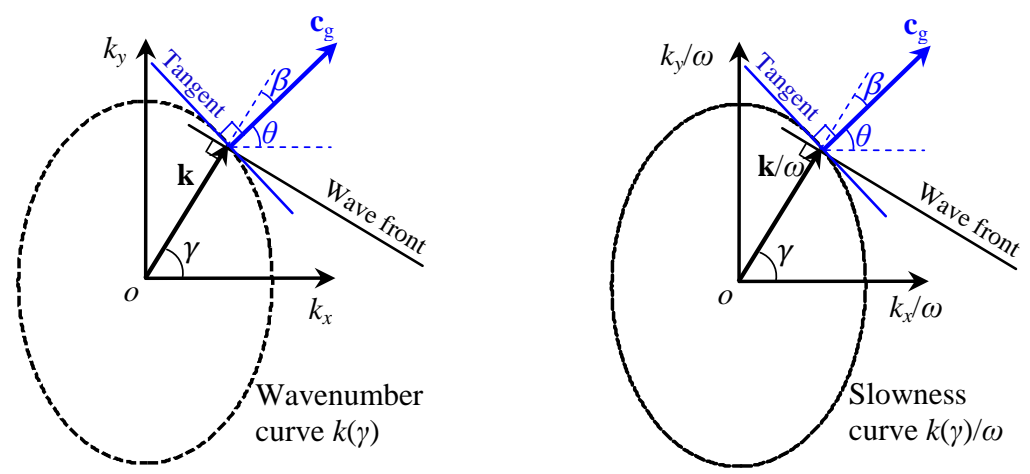

Figure 1 Schematics of geometric relations of GW in composite laminates: (a) the geometric relation of GW (in the far field) generated by sources at different locations; (b) wavenumber and slowness curves. The geometric relation between the wavenumber vector $\mathbf{k}$ and the group velocity vector $\mathbf{c}_{\mathrm{g}}$ is given in figure $\mathrm{b}$. 


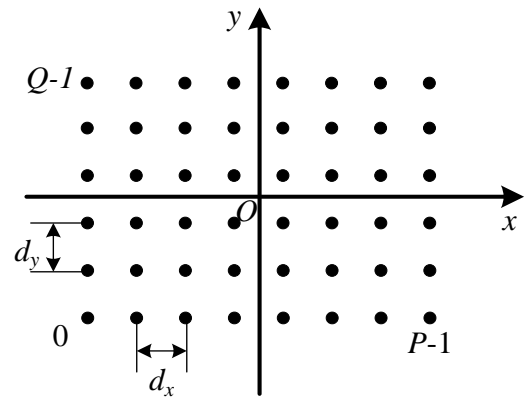

Figure 2 Schematic of a $P \times Q$ rectangular gird array 

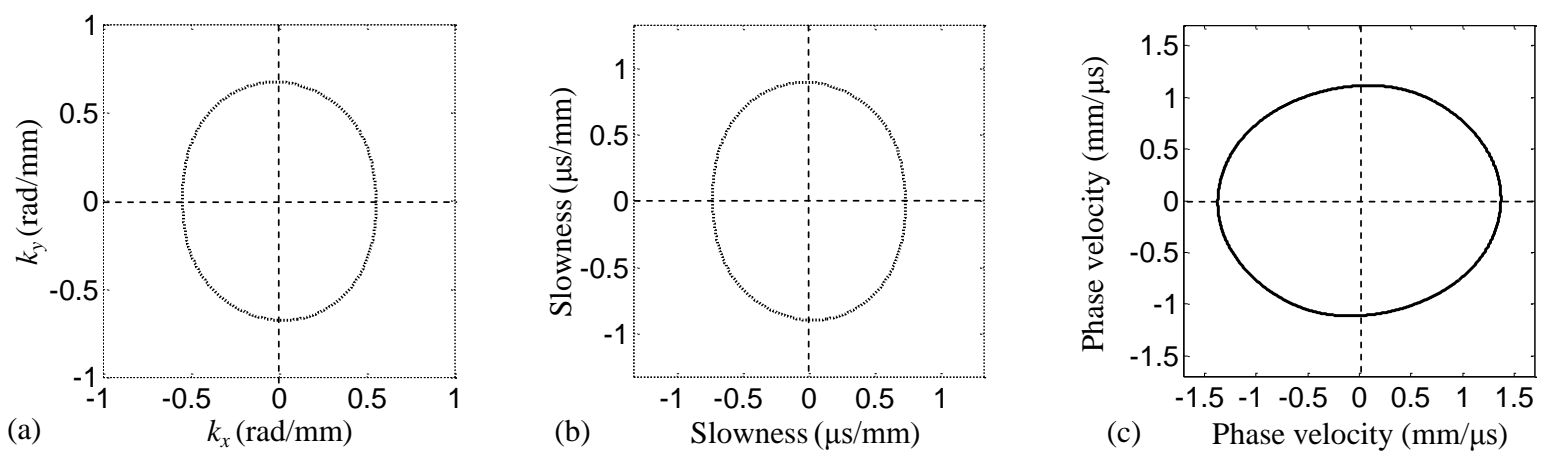

Figure 3 (a) wavenumber, (b) slowness and (c) phase velocity plots of the $120 \mathrm{kHz} \mathrm{A}_{0}$ mode in the $[0 / 45 / 90 /-45]_{s}$ CFRP composite plate. 

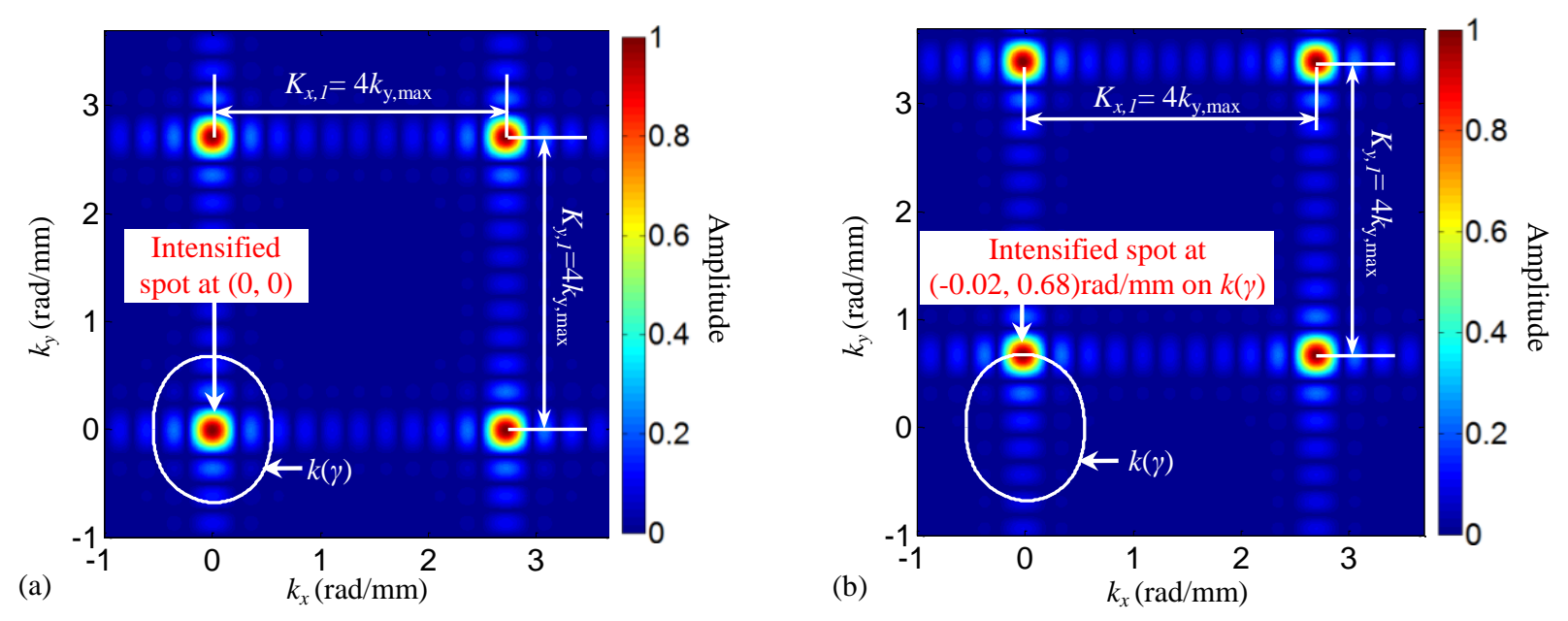

Figure $4 \quad B F\left(\mathbf{k} \mid w_{p, q}, \theta_{S}\right)$ images for the array A1: (a) $B F\left(\mathbf{k} \mid w_{p, q}=1\right.$, no) without phase delays, and (b) $B F\left(\mathbf{k} \mid w_{p, q}=1, \theta_{S}=90^{\circ}\right)$ with phase delays of $(-0.02,0.68) \cdot \mathbf{p}_{p, q}$. The solid white curve is the wavenumber curve $k(\gamma)$ of the $120 \mathrm{kHz} \mathrm{A}_{0}$ mode in the [0/45/90/-45] $]_{\mathrm{s}}$ CFRP composite plate. 

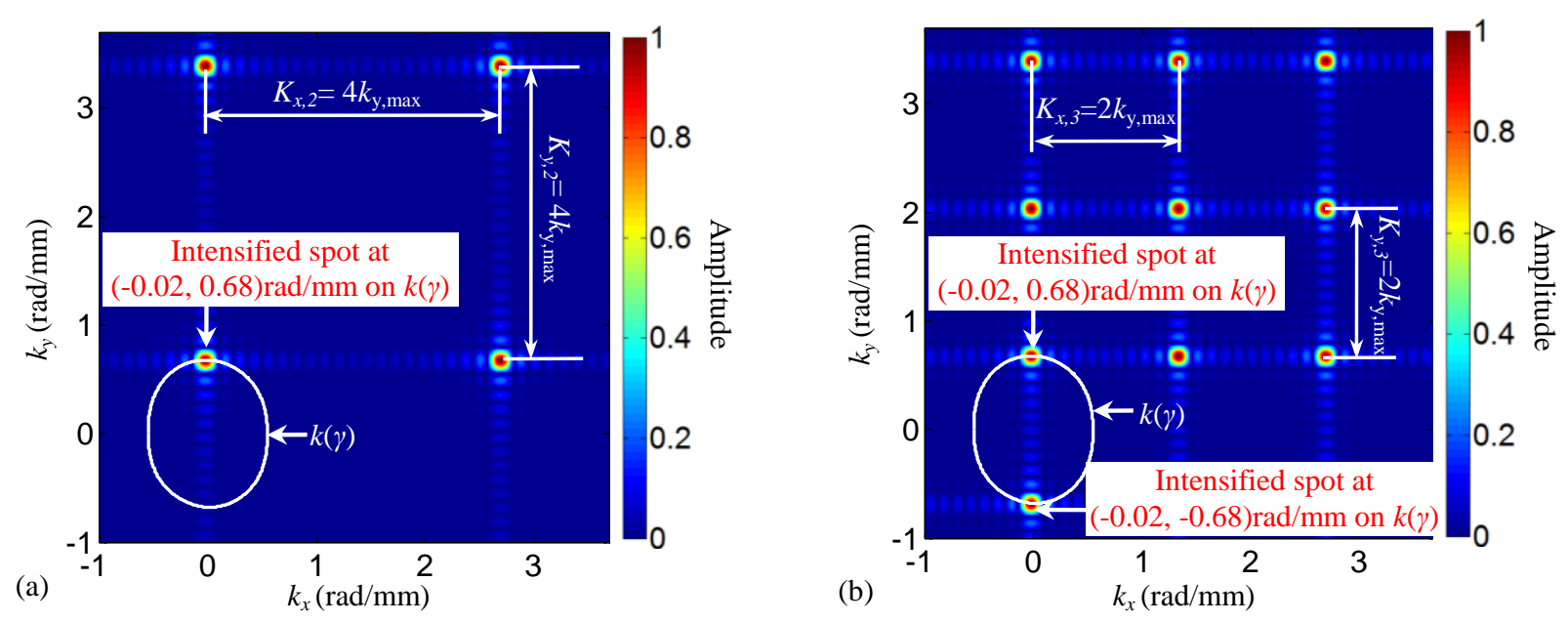

Figure $5 \quad B F\left(\mathbf{k} \mid w_{p, q}=1, \theta_{S}=90^{\circ}\right)$ images when phase delays $(-0.02,0.68) \cdot \mathbf{p}_{p, q}$ are applied: (a) for array $\mathrm{A} 2$, and (b) for array A3. The solid white curve is the wavenumber curve $k(\gamma)$ of the $120 \mathrm{kHz}$ $\mathrm{A}_{0}$ mode in the $[0 / 45 / 90 /-45]_{\mathrm{s}}$ CFRP composite plate. 
(a)

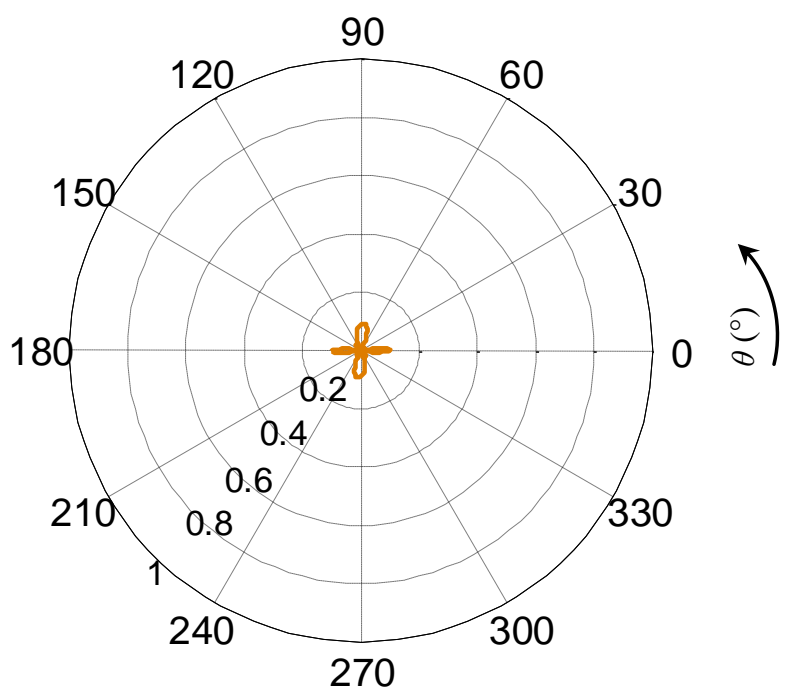

(b)
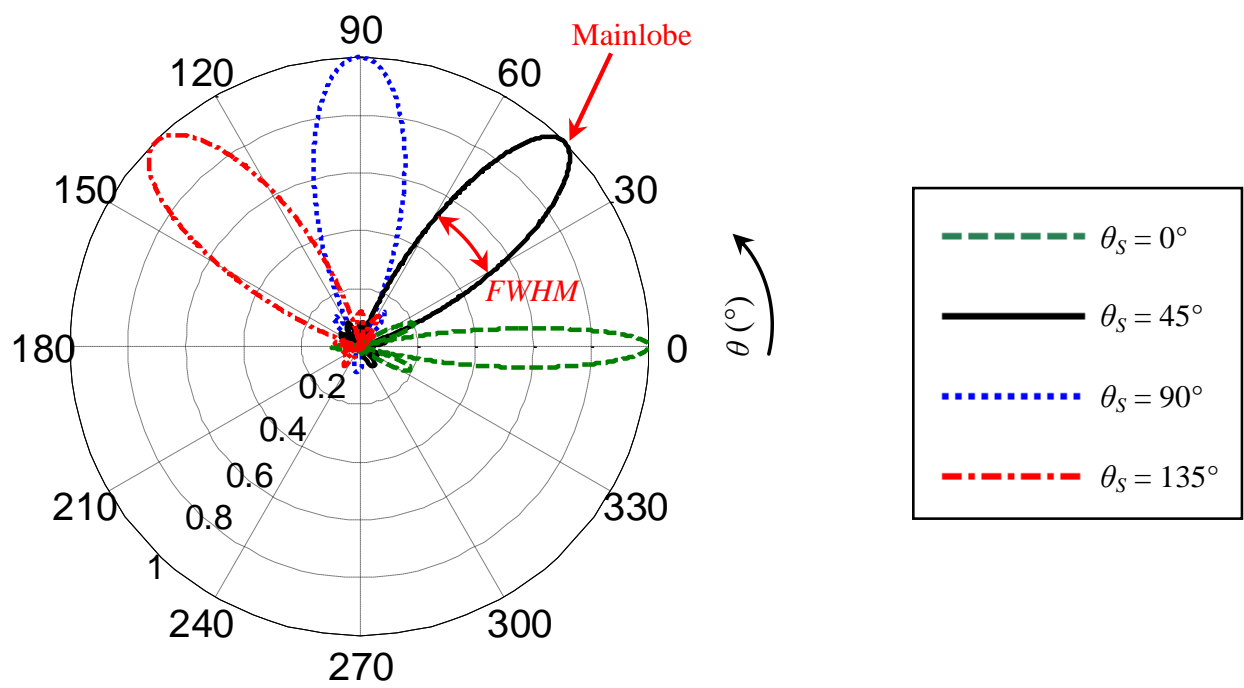

Figure $6 \quad B F\left(\theta \mid w_{p, q}, \theta_{S}\right)$ plots for array A1: (a) $B F\left(\theta \mid w_{p, q}=1\right.$, no) plot without beamsteering, and (b) $B F\left(\theta \mid w_{p, q}=1, \theta_{S}\right)$ plots when beamsteering angles are $\theta_{S}=0^{\circ}, 45^{\circ}, 90^{\circ}$ and $135^{\circ}$. 
(a)

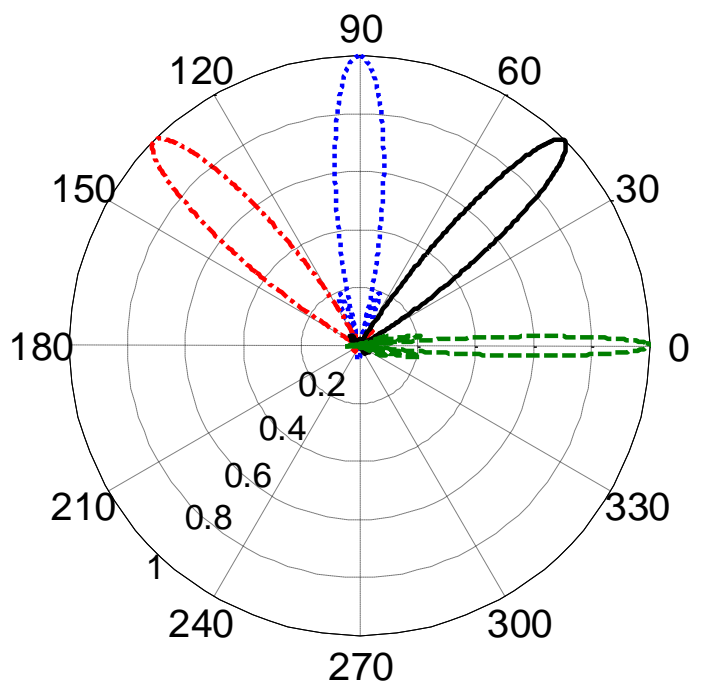

$\underset{0}{\stackrel{5}{c}}$
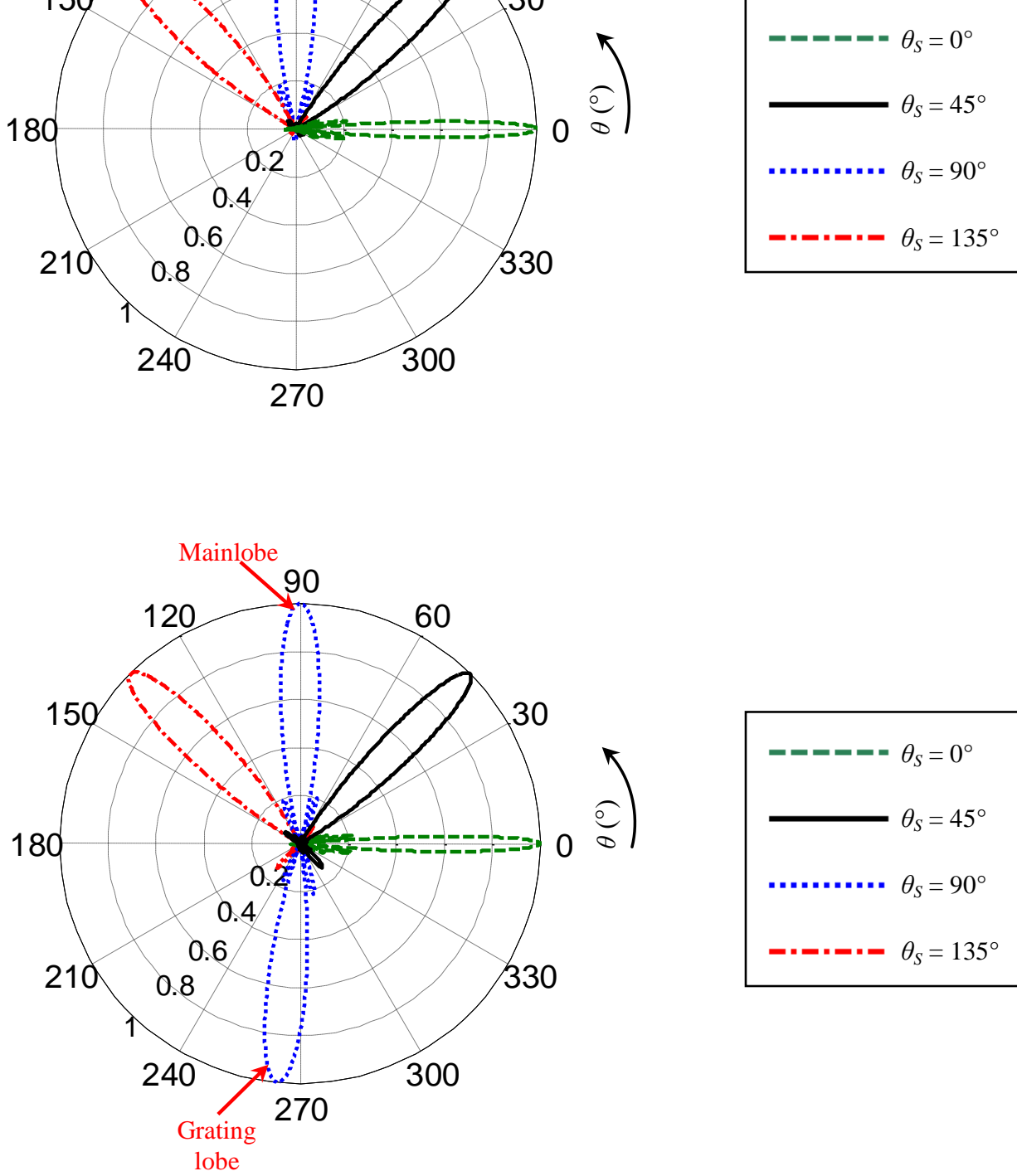

ฮ

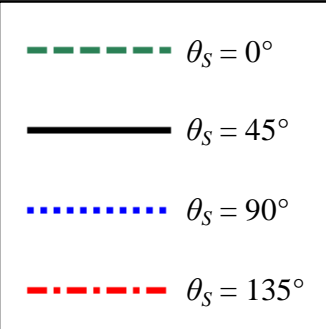

Figure $7 \quad B F\left(\theta \mid w_{p, q}=1, \theta_{S}\right)$ plots when beamsteering angles are $\theta_{S}=0^{\circ}, 45^{\circ}, 90^{\circ}$ and $135^{\circ}$ : (a) for array $\mathrm{A} 2$, and (b) for array A3. 

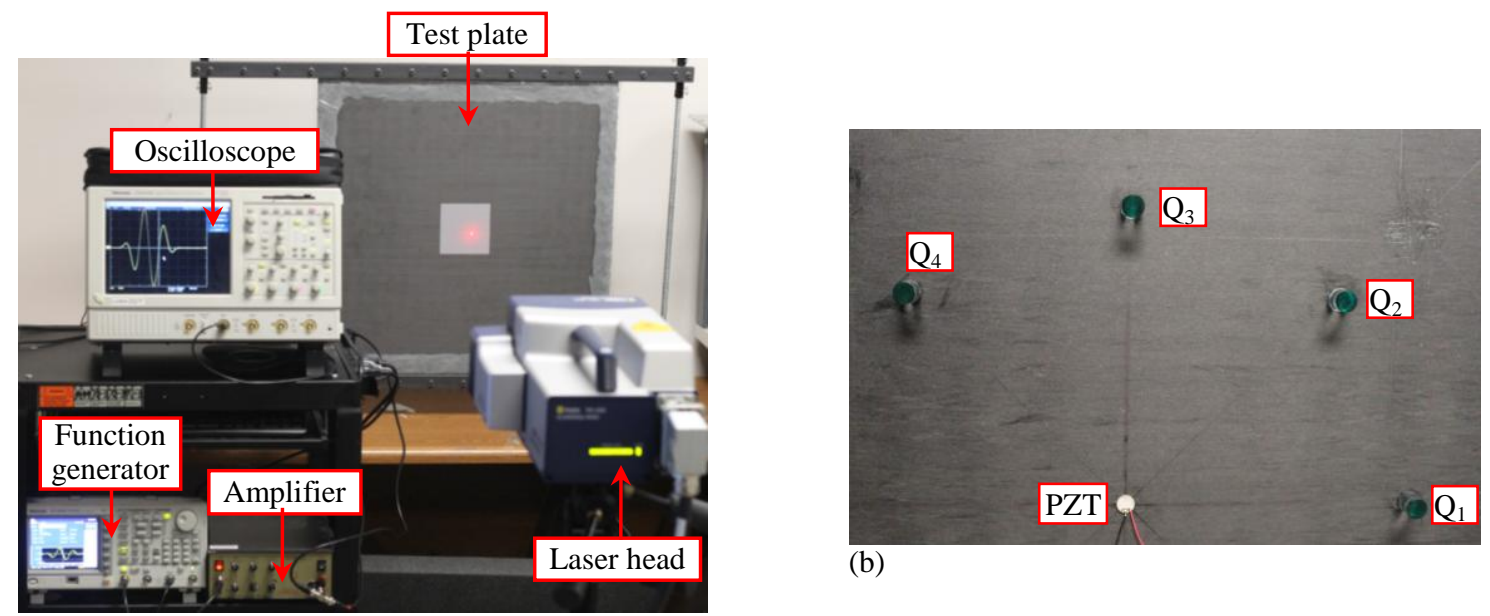

(b)

(a)

$\mathrm{Q}_{3}$

(c)

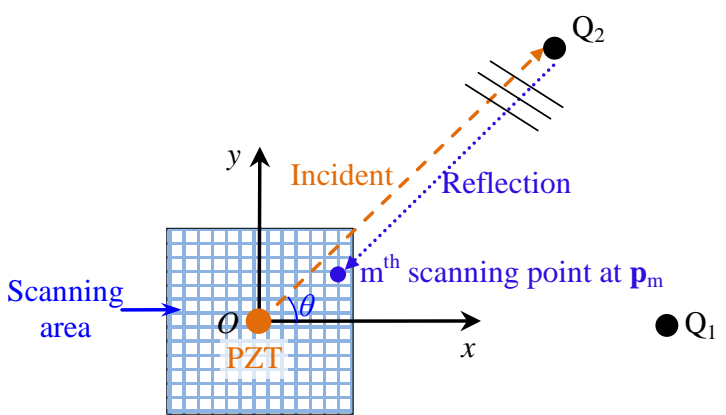

Figure 8. Experimental setup for the detection of multiple defects in a CFRP composite laminate: (a) photo of test setup, (b) front side of the test specimen, and (c) schematic of the sensing layout. 

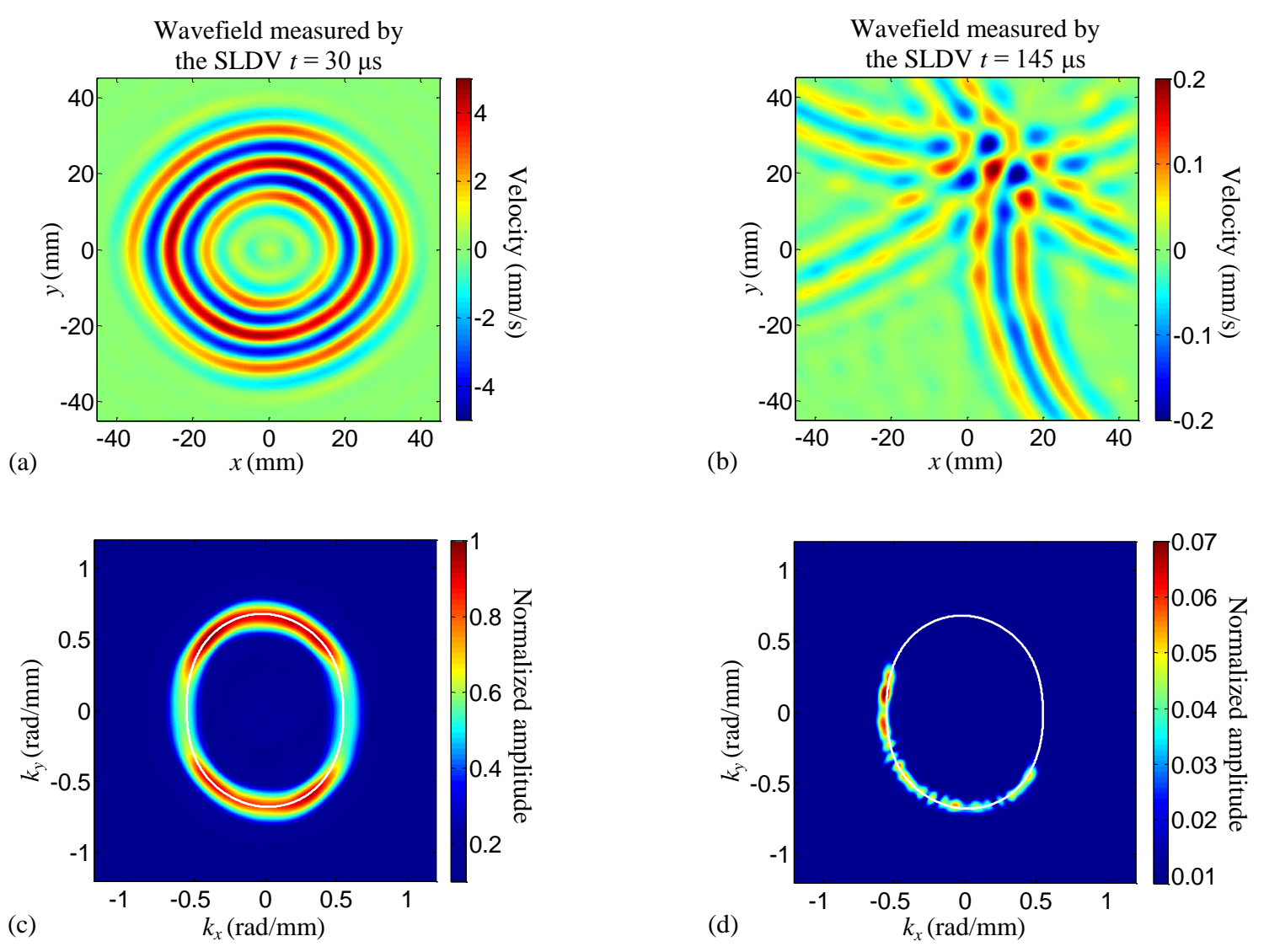

Figure 9. SLDV measurements: (a) and (b) are wavefields in the $45 \mathrm{~mm} \times 45 \mathrm{~mm}$ scanning area at $30 \mu \mathrm{s}$ showing incident waves and at and $145 \mu$ s showing reflection waves, respectively; (c) and (d) are wavenumber spectra of incident and reflection waves at $120 \mathrm{kHz}$. The solid white line is the wavenumber curve of the $\mathrm{A}_{0}$ mode at $120 \mathrm{kHz}$. 

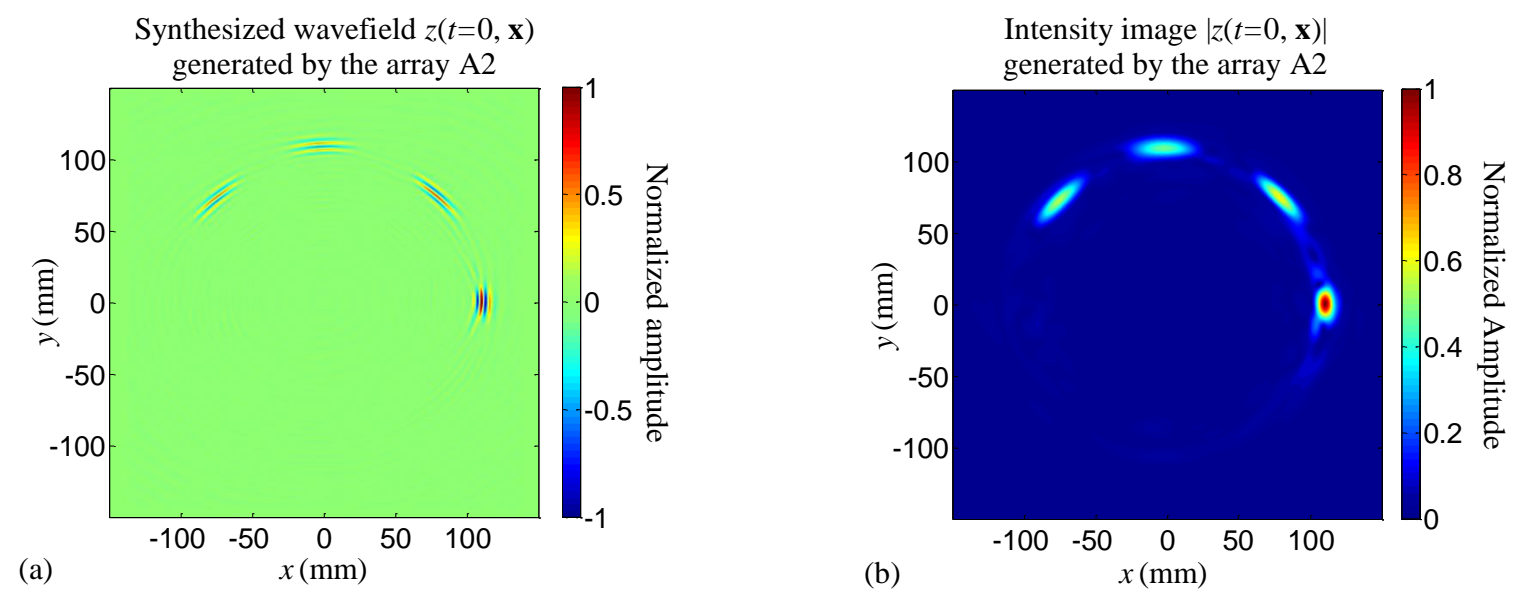

Figure 10. Phased array imaging results for array A2: (a) synthesized wavefield $z(t=0, \mathbf{x})$, and (b) intensity image $|z(t=0, \mathbf{x})|$. 

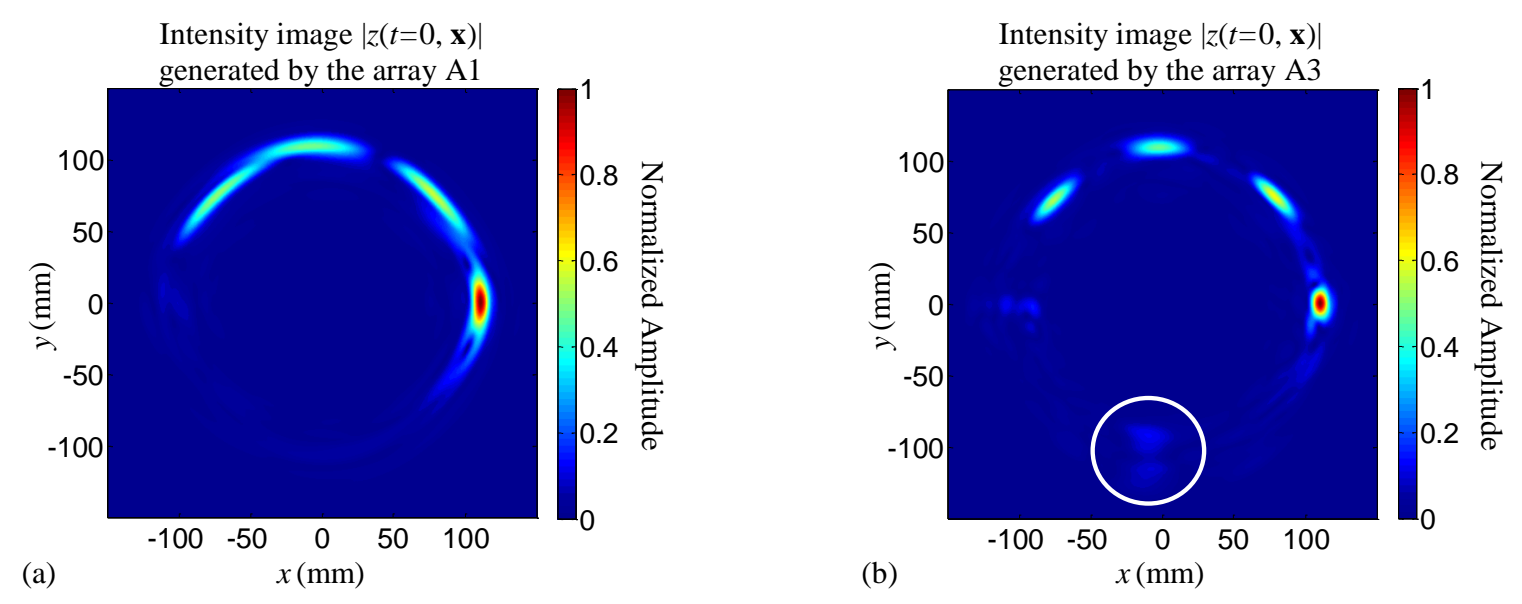

Figure 11. Phased array imaging results: (a) for array A1, and (b) for array A3. 
Table 1 Material properties of a single ply.

\begin{tabular}{ccccccccccc}
\hline $\begin{array}{c}\rho \\
\left(\mathrm{kg} / \mathrm{m}^{3}\right)\end{array}$ & $\begin{array}{c}\mathrm{E}_{1} \\
(\mathrm{GPa})\end{array}$ & $\begin{array}{c}\mathrm{E}_{2} \\
(\mathrm{GPa})\end{array}$ & $\begin{array}{c}\mathrm{E}_{3} \\
(\mathrm{GPa})\end{array}$ & $\begin{array}{c}\mathrm{G}_{12} \\
(\mathrm{GPa})\end{array}$ & $\begin{array}{c}\mathrm{G}_{13} \\
(\mathrm{GPa})\end{array}$ & $\begin{array}{c}\mathrm{G}_{23} \\
(\mathrm{GPa})\end{array}$ & $\nu_{12}$ & $\nu_{13}$ & $\nu_{23}$ & $\begin{array}{c}\text { Thickness } \\
(\mathrm{mm})\end{array}$ \\
\hline 1577.8 & 129.6 & 8.7 & 8.7 & 4.8 & 4.8 & 2.9 & 0.30 & 0.30 & 0.34 & 0.3175 \\
\hline
\end{tabular}


Table 2 Configurations of three rectangular arrays for beamforming study

\begin{tabular}{lccc}
\hline & $\begin{array}{c}\text { Number of elements } \\
P \times Q\end{array}$ & $\begin{array}{c}\text { Spacing } \\
d(\mathrm{~mm})\end{array}$ & $\begin{array}{c}\text { Span } \\
D(\mathrm{~mm})\end{array}$ \\
\hline Array A1 & $11 \times 11$ & $d_{x, 1}=d_{y, 1}=\lambda_{\mathrm{y}, \min } / 4=2.3$ & $D_{x, 1}=D_{y, 1}=23$ \\
Array A2 & $21 \times 21$ & $d_{x, 2}=d_{y, 2}=\lambda_{\mathrm{y}, \min } / 4=2.3$ & $D_{x, 2}=D_{y, 2}=46$ \\
Array A3 & $11 \times 11$ & $d_{x, 3}=d_{y, 3}=\lambda_{\mathrm{y}, \min } / 2=4.6$ & $D_{x, 3}=D_{y, 3}=46$ \\
\hline
\end{tabular}


Table $3 \quad B F\left(\mathbf{k} \mid w_{m}, \theta_{S}\right) \quad$ characteristics of the three rectangular grid arrays

FWHM $(\mathrm{rad} / \mathrm{mm}) \quad$ Wavenumber periods, $K(\mathrm{rad} / \mathrm{mm})$

\begin{tabular}{lll}
\hline Array A1 & $F W H M_{x, I}=F W H M_{y, l}=0.30$ & $K_{x, I}=K_{y, 1}=2 \pi / d_{x, I}=2 \pi / d_{y, l}=4 k_{y, \min }=2.72$ \\
Array A2 & $F W H M_{x, 2}=F W H M_{y, 2}=0.15$ & $K_{x, 2}=K_{y, 2}=2 \pi / d_{x, 2}=2 \pi / d_{y, 2}=4 k_{y, \min }=2.72$ \\
Array A3 & $F W H M_{x, 3}=F W H M_{y, 3}=0.15$ & $K_{x, 3}=K_{y, 3}=2 \pi / d_{x, 3}=2 \pi / d_{y, 3}=2 k_{y, \min }=1.36$ \\
\hline
\end{tabular}


Table $4 F W H M$ values of directional beam patterns $B F\left(\theta \mid w_{p, q}, \theta_{S}\right)$ at different directions of $\theta_{S}$.

\begin{tabular}{ccccc}
\hline & $\theta_{S}=0^{\circ}$ & $\theta_{S}=45^{\circ}$ & $\theta_{S}=90^{\circ}$ & $\theta_{S}=135^{\circ}$ \\
\hline Array A1 & $14^{\circ}$ & $31^{\circ}$ & $35^{\circ}$ & $35^{\circ}$ \\
Array A2 & $7^{\circ}$ & $16^{\circ}$ & $18^{\circ}$ & $18^{\circ}$ \\
Array A3 & $7^{\circ}$ & $16^{\circ}$ & $18^{\circ}$ & $18^{\circ}$ \\
\hline
\end{tabular}

